\title{
Associação entre o excesso de peso e de gordura corporal nas crianças com sobrepeso/obesidade materno
}

\author{
Mariana de Santis Filgueiras, Fernanda Martins de Albuquerque, Ana Paula Pereira Castro, Naruna \\ Pereira Rocha, Luana Cupertino Milagres, Juliana Farias de Novaes
}

\begin{abstract}
Resumo
A obesidade infantil é um problema de saúde pública global, sendo que no Brasil sua prevalência é de 14,3\% em crianças de 5 a 9 anos de idade. O ambiente familiar exerce um papel fundamental na gênese da obesidade, sendo responsável pela formação de hábitos alimentares e de estilo de vida. Tendo em vista essa relação, é importante avaliar o estado nutricional dos pais, sobretudo das mães, uma vez que estas tendem a influenciar a formação destes hábitos. Avaliar a associação entre a presença de excesso de peso/gordura corporal em crianças e o estado nutricional materno. Estudo transversal com 292 crianças de 8 e 9 anos matriculadas em escolas públicas e privadas na zona urbana do município de Viçosa-MG. Foram aferidos peso e estatura para o cálculo do Índice de Massa Corporal por Idade (IMC/I) de acordo com a Organização Mundial da Saúde (OMS, 2007). A gordura corporal $(\% \mathrm{GC})$ foi obtida pela bioimpedância bipolar (Tanita ${ }^{\circledR}$ Modelo Ironman BC 553) classificada segundo Lohman (1992). Para avaliar o estado nutricional materno, foram aferidas as medidas de peso e estatura para o cálculo do IMC, utilizando os pontos de corte da OMS (2000). Crianças e mães com sobrepeso e obesidade foram classificadas como excesso de peso. As análises estatísticas foram realizadas no software SPSS versão 20, sendo considerado o nível de significância de 5\%. Este estudo foi aprovado pelo Comitê de Ética em Pesquisa com Seres Humanos da Universidade Federal de Viçosa. As prevalências de crianças com excesso de peso e de gordura corporal foram de 32,8\% e 49,7\%, respectivamente, e 57,5\% das mães apresentaram excesso de peso. As prevalências de crianças com excesso de peso e de gordura corporal foram maiores entre aquelas com mães com excesso de peso em comparação às mães sem excesso peso $(42,9 \%$ versus $18,5 \%, 55,4 \%$ versus $42,7 \%, \mathrm{p}<0,05)$. Foram observadas correlações significantes entre os dados das crianças (IMC, escore-Z de IMC/I e \%GC) com o IMC materno (IMC: 0,351; $\mathrm{p}<0,001$; escore-Z de IMC/I: 0,338; $\mathrm{p}<0,001 ; \% \mathrm{GC}: 0,317, \mathrm{p}<0,001)$. As prevalências de excesso de peso e de gordura corporal em crianças estiveram associadas ao excesso de peso materno. Resultados como esses ressaltam a importância de elaborar estratégias educativas relacionadas à saúde para crianças juntamente aos seus pais, considerando sua importância na formação de hábitos alimentares e de estilo de vida, de modo a prevenir o surgimento de complicações cardiometabólicas na infância e na fase adulta.
\end{abstract}

Descritores: Obesidade Pediátrica; Adiposidade; Saúde da Família. 